\title{
Comunidades de aprendizaje móvil, MasteryLearning y el Problema 2 sigma como estrategias para el conocimiento previo, en la resolución de actividades \\ Mobile Learning Communities, Mastery Learning and the 2 Sigma Problem as strategies for prior knowledge in activity resolution. \\ Comunidades de aprendizagem móvel, Aprendizagem de domínio e Problema 2 sigma como estratégias de conhecimento prévio, na resolução de atividades \\ Carlos Luis Gómez - Valderrama, Rosario Haydeé Ramírez - Sánchez *
}

\author{
Profesor Mención Informática- Universidad Pedagógica Experimental Libertador. UPEL-IMPM, Trujillo, Venezuela. (D) \\ Doctora en Educación. Universidad Pedagógica Experimental Libertador. UPEL-IMPM, San Cristobal, Venezuela. (D)
}

Forma de citar: Gómez, C., Ramírez, R. (2016). Comunidades de aprendizaje móvil, Mastery Learning y el Problema 2 sigma como estrategias para el conocimiento previo, en la resolución de actividades. Encuentro Internacional en Educación Matemática Versión ISSN 2539-1885. La Educación Matemática como Herramienta en el Desempeño Profesional Docente. Cúcuta, Colombia. 25 - 32

Resumen: Esta experiencia es parte de una investigación sobre el futuro de las tecnologías, dentro y fuera del salón de clases; se basa en una investigación acción, con la finalidad de planificar, actuar, observar y reflexionar (Kemmis ,1989 ),para transformar la situación de partida e innovar en nuevos espacios de enseñanza-aprendizaje que indaguen estrategias mediadas por tecnologías, en la resolución de actividades por parte de los participantes del curso La web, un espacio para el aprendizaje, edición UPEL-IMPM-Táchira. Se partió de las ideas de Bloom (1968 y 1984 ) acerca de la estrategia instruccional Mastery Learning y la tutorización (Problema 2 sigma), respectivamente, a través de una comunidad de aprendizaje (Fidalgo , 2014 ) en la que el grupo de personas que aprenden utilizan herramientas comunes en un mismo entorno, que puede ser un teléfono móvil (WhatsApp) o unas redes sociales, tales como Facebook o Twitter. La muestra estuvo compuesta por cuarenta (40) profesores de pregrado y postgrado de diferentes especialidades, algunos de los cuales tienen competencias digitales. Cuando se concluyó, se determinó que la puesta en marcha de discusiones grupales en una comunidad de aprendizaje móvil, con herramientas tales como Whats App, apoyadas en grupos de Facebook y tutorías en línea, como estrategia anticipada a la resolución de las actividades en cada unidad temática, logró una mayor comprensión de los contenidos, obteniéndose un $90 \%$ de conocimientos previos óptimos de los participantes, lo que permitió afrontar y realizar las tareas planteadas. A su vez, se personalizó el aprendizaje, al hacerlo adaptativo y flexible, para realizar el análisis del aprendizaje, luego de cuatro (4) semanas dentro del aula virtual, con la propuesta de otras tutorías en línea, para aquellos participantes con actividades atrasadas, por lo que se logró $85 \%$ de recuperación; ello permitió una finalización exitosa del curso.

Palabras clave Comunidad de aprendizaje, MasteryLearning, problema 2 sigma, WhatsApp.

\begin{abstract}
This experience forms part of an investigation that addresses the future of technologies, within and beyond the classroom; it is based on an action research whose goal is to plan, act, observe and reflect (Kemmis, 1989). Said research also seeks to transform the initial situation and break ground in new teaching-learning spaces that explore strategies mediated by technologies, in the resolution of activities by participants of the course: "The Web, a space for learning, UPEL-IMPM-Táchira edition.” Taken as a point of departure were Bloom's ideas $(1968,1984)$ pertaining to the Mastery Learning instructional strategy and tutoring (Sigma 2 Problem), respectively, through a learning community (Fidalgo, 2014), in which the group of learners uses common tools (a smartphone with WhatsApp or social networks like Facebook and Twitter) in a single environment. The sample was composed of 40 undergraduate and graduate professors belonging to various disciplines, some possessing digital competences. By the end of the study, it was determined that initiating group discussions in a mobile learning community with tools like WhatsApp, supported by Facebook groups and online tutorials as a strategy designed for the completion of activities within each thematic unit, led to a greater understanding of the contents, obtaining $90 \%$ of participants' previous optimum knowledge and enabling them to confront and complete given tasks. Furthermore, learning was personalized by making it adaptive and flexible, in order to complete its analysis, after four (4) weeks inside the virtual classroom, with the proposal of other online tutorials and for those participants with overdue activities. Consequently, an $85 \%$ recovery was achieved; this facilitated the course's successful conclusion.
\end{abstract}

Keywords Learning community, Mastery Learning, Sigma 2 Problem, WhatsApp.

* Autor para correspondencia rosario@ciegc.org.ve

2590-9215 C 2017 Universidad Francisco de Paula Santander. Este es un artículo bajo la licencia CCBY 
Resumo: Essa experiência faz parte de uma pesquisa sobre o futuro das tecnologias, dentro e fora da sala de aula; baseia-se em uma pesquisa ação, com o objetivo de planejar, agir, observar e refletir (Kemmis, 1989), transformar a situação inicial e inovar em novos espaços de ensino-aprendizagem que investiguem estratégias mediadas por tecnologias, na resolução de atividades pelos participantes do curso "A Web, um espaço para aprender, UPEL-IMPM-Táchira". Começando com as ideias de Bloom (1968 e 1984) sobre a estratégia instrucional de Aprendizagem de Mestria e a tutoria (Problema 2 Sigma), respectivamente, através de uma comunidade de aprendizagem (Fidalgo, 2014), onde o grupo de pessoas usa ferramentas comuns em um mesmo ambiente, que pode ser um smartphone (WhatsApp) ou algumas redes sociais, como Facebook e Twitter. A amostra foi composta por 40 professores de graduação e pós-graduação de diferentes especialidades, alguns dos quais possuem competências digitais. Ao final do estudo, ficou determinado que iniciar discussões em grupo em uma comunidade de aprendizado móvel, com ferramentas como o WhatsApp, apoiado por grupos do Facebook e tutoriais online, como estratégia antecipada para a resolução de atividades em cada unidade temática, compreensão dos conteúdos, obtendo $90 \%$ do conhecimento prévio ótimo dos participantes, que permitiu enfrentar e realizar as atividades levantadas. Além disso, o aprendizado foi personalizado, tornando - o adaptativo e flexível para a realização de análises de aprendizagem, após 4 (quatro) semanas dentro da sala de aula virtual, com a proposição de outros tutoriais online, para os participantes com atividades vencidas de recuperação foi alcançado; isso tornou possível a conclusão bem-sucedida do curso.

Palabras clave Comunidad de aprendizaje, MasteryLearning, problema 2 sigma, WhatsApp.

\section{Introducción}

Al indicar acerca de conocimientos previos, Piaget (1968) sostiene que el entendimiento de nuevas ideas, al ser escaso o confuso, puede crear barreras para el aprendizaje, porque se asimila la información sin comprender la; de allí que, este entendimiento es indispensable para la asimilación y acomodación de la información, lo que acarrea dificultades en su posterior adaptación para que dé cabida al nuevo saber.

Se olvida, al poco tiempo, la información recibida, independientemente de los procesos formativos en los distintos niveles y modalidades de educación, los cuales están considerando cambios que requieren reajustes en las praxis pedagógicas, conocimientos y perspectiva de enseñanza, en relación con los nuevos ambientes de aprendizaje, inclusive los mediados por tecnologías.

Esto dilucida que, de acuerdo con Ausubel citado por Ontoria y otros (2006): "La adquisición de información nueva depende en alto grado de las ideas pertinentes que ya existen en la estructura cognitiva.” (p. 22).Además afirma el autor, que el estar familiarizado con el material en cuestión tiene un efecto positivo mayor que con el desconocido.

El aprendizaje, en las personas, ocurre a través de la nueva información y la conocida en la estructura cognitiva; no obstante, los nuevos espacios de aprendizaje del siglo XXI, especialmente el digital, lo complicarían aún más, porque las nuevas tendencias dicen, según Siemens (2004) que el aprendizaje puede residir fuera de nosotros mismos, porque ocurre en una variedad de formas - a través de comunidades de práctica, redes personales. Ello está en concordancia con lo que plantea Vygotsky (1978) quien señala que existe la mediación social debido a que: "En el desarrollo cultural...toda función aparece dos veces: primero, a nivel social, y más tarde, a nivel individual”. (p. 94).
El autor esboza que existen dos (2) formas de aprender, primero entre las personas (plano interpersonal) y posteriormente, en el interior del propio yo (plano intrapersonal); por lo que se asimila en interacción con otros y se produce cuando, intrínsecamente, las nuevas ideas corresponden con ideas relevantes. Por otra parte, estudios diversos han demostrado que la disposición de los estudiantes frente al aprendizaje, así como su capacidad cognitiva, sus habilidades y estrategias son fundamentales para iniciar el aprendizaje de un nuevo contenido, por lo que el concepto de conocimientos previos, conduce hacia los objetivos de aprendizaje y a sus estrategias, así como a la intervención del mediador del proceso de enseñanza y aprendizaje.

De allí que el resultado de la interiorización de los procesos de mediación desarrollados por nuestra cultura y a través de ella, ha dado cabida a la incorporación de la tecnologías en los sistemas educativos; Las universidades están incursionando en el aprendizaje electrónico. Es decir, E-Learning, donde "las interacciones son analizadas e interpretadas, en su mayoría, desde el enfoque de la escuela histórico-cultural de Vygotsky", de acuerdo con lo indicado por Pérez (2009) , además de que "...describen el valor de las interacciones y su relación con las zonas de desarrollo próximo, como una red social de interrelaciones y construcción colectiva del conocimiento". En este sentido, estaría en relación con lo planteado por Wenger, citado por García (2002) , quien afirma que "...desde el principio de la historia, los seres humanos han formado comunidades que acumulan su aprendizaje colectivo en prácticas sociales...”; es decir, en una comunidad de aprendizaje. Fidalgo (2014) manifiesta que "una red social puede ser perfectamente una comunidad"; además, alega que podría ser definida como un grupo de personas que aprende en común, utilizando herramientas habituales en un mismo entorno; es de interés común la obtención de conocimientos previos para el desarrollo y que sean afines a sus intereses profesionales o personales. 
Además, el autor argumenta que una red social en la cual existen intereses comunes sobre un tipo de conocimiento, puede ser justamente una comunidad de aprendizaje.

En este contexto, la Asociación Universitaria de Formación del Profesorado, AUFOP (2010), señala que: "las comunidades de aprendizaje transforman el contexto, por ejemplo, mediante la creación de grupos heterogéneos dentro del aula". (p. 12).Estos grupos, que pueden ser interactivos, dinamizan las interacciones del grupo, porque crean colaboración, cooperación y solidaridad entre los estudiantes, con el fin de que todos afronten con éxito las actividades.

Por otra parte, según Vygotsky (1978) el aprendizaje está condicionado por la sociedad en la que nacen y se desarrollan las personas; se deduce que la clave del aprendizaje, dentro de las comunidades de aprendizaje, es el dialógico, así como las interacciones que se producen dentro de ellas.

En ese mismo sentido, el tutor podría aprovechar dichas interacciones para propiciar a través de preguntas problematizadoras, socráticas, afirmativas, entre otras, discusiones grupales en tiempo real con evaluación formativa, así como métodos modernos mediante el uso de instrumentos móviles, tales como, los computadores portátiles, las tabletas informáticas, los teléfonos inteligentes (smartphones) y los teléfonos móviles.

Barbera, Badia y Momimó, citados por Pérez (2009, p. 1), exponen que "...las interacciones y la comunicación es el contexto virtual de aprendizaje, pues en él surgen una serie de relaciones sociales y afectivas en torno al aprendizaje mediado por la tecnología".

En cambio, Meza (2012), afirma que "el E-Learning genera cambios en la forma de gestionar la educación" (p. 10); distingue que esta modalidad, conlleva ciertas dificultades que hay que tener en cuenta para resolver, en cuanto al diseño, los cursos y los materiales, la evaluación, a través del uso de las tecnologías disponibles y de la forma de moderar el proceso de prendizaje.

De allí la importancia en la forma de moderar al grupo, por cuanto el mediador debe asumir varios roles: ser dinamizador, asesor y facilitador dentro de los espacios digitales, con la finalidad de obtener un ambiente acorde con las actividades planificadas, para que se cumplan y eleven la calidad y productividad del proceso de enseñanza y aprendizaje. Ello permite la motivación de las interacciones ser el "impulso de obtener éxito y destacar. ...de establecer objetivos importantes, apuntando a la excelencia, con un enfoque en el trabajo bien realizado..."Pacheco-Carrascal (2016, p. 150) citando a McClellan (1961).
Permitiendo "extender el acceso a las oportunidades educativas... al implementar estrategias de enseñanza flexibles... y que, en consonancia, ofrecen diferentes alternativas de acceso al conocimiento" (González 2015, p. 108) . Pudiéndose aplicar el problema 2 sigma, propuesto por Bloom (1984), a través del cual se "idean las condiciones bajo tutoría para alcanzar altos niveles de logros en estudiantes... durante el proceso de enseñanza-aprendizaje".

Se puede centrar en la realimentación y en el aprendizaje individualizado, respetando el ritmo de los discentes y recibiendo reinformación inmediata sobre los avances de conocimientos adquiridos; para minimizar la "...desmotivación, baja participación y el no cumplimiento de los compromisos por parte del estudiante, conllevan a hacer una reflexión ...y un cambio en las prácticas del docentes en las que se utilicen diversos escenarios, recursos(lúdicos y tecnológicos) que sirvan como mediadores y permitan mejorar la relación docente - estudiantes" (Pabón-Gómez 2014, p. 38), al aplicar estrategias basadas en grupos, individualizadas, de enseñanza y aprendizaje basado en que los estudiantes van a lograr un alto nivel de comprensión en un dominio determinado si se les da tiempo suficiente.

Por otra parte, Bloom en el año 1968 en su propuesta acerca de una estrategia denominada por él como "Aprendizaje para el dominio" o MasteryLearning, sugiere que para aprender un material determinado y conseguir un nivel alto de dominio y conocimiento, el profesional de la docencia debería en su "instrucción permitir el tiempo requerido" (Bloom 1984, p. 3 ) para que los diferentes estudiantes puedan aprender.

De igual manera, el autor precitado señala que los estudiantes no pueden participar activamente en el aprendizaje, si la instrucción es pobre ya que son incapaces de comprender lo que se les enseña y lo que tienen que hacer.

No se puede olvidar la época actual de la humanidad, signada por la sociedad de la información y del conocimiento, que implica que la tutorización podría aprovechar las conexiones proporcionadas entre el mediador-estudiantes y estudiante-estudiante, a través de la interacción con el medio y una comunidad de aprendizaje, ya sea con herramientas digitales tales como Facebook, Google + o con aplicaciones móviles como WhatsApp. Ello fomentaría el autoaprendizaje (intrapersonal) que da lugar a que el estudiante se responsabilice de la construcción de su propio aprendizaje, pasando primero por el aprendizaje social (interpersonal) con otros y de otros.

De allí que, se puede sustentar el aprendizaje mediado por las tecnologías, por intermedio de la teoría socio-cultural de Vygotsky, en la cual lo nuevo se suma a lo conocido (conocimiento previo); es decir, el aprendizaje doble a saber, la interacción social y el desarrollo cognitivo. 
De igual manera, para lograr la instrucción individualizada, el mediador debería suprimir algunas actividades grupales que permitan la tutoría uno a uno o en grupos pequeños, pudiéndose aplicar el problema 2 sigma de Bloom, en los estudios a distancia, a través de mecanismos diferentes proporcionados por internet, tales como las videoconferencias a través de Hangouts, Skype, entre otros. Ello permitiría la motivación de los participantes, para que tengan la oportunidad de corregir sus errores, de acuerdo con su avance en sus estudios; la interacción y realimentación del profesor será una ayuda necesaria, para que el estudiante interactúe eficazmente con el material didáctico y se obtengan los objetivos y metas del aprendizaje.

En consecuencia, se deben plantear estrategias de aprendizaje que no solamente dependan del estudiante, sino también de la calidad de la enseñanza de vanguardia que disponga de ambientes de aprendizaje digital. Farnos (2015) afirma que: "Las aulas evolucionarán hacia comunidades abiertas, flexibles y centradas en el alumno". Ello comienza con la interacción en redes de personas que comparten el conocimiento tácito que poseen los individuos, con el fin de ampliarlo en comunidades de aprendizaje digitales, lo cual se convierte en conocimiento explícito, que adopta la forma de un concepto nuevo, que permite los participantes su aporte en soluciones para las actividades propuestas dentro de un ambiente virtual de aprendizaje.

De lo antes formulado, surge de la necesidad de incorporar cambios en la forma de la enseñanza a través de los estudios a distancia, que se centre en el aprendizaje constructivo y experiencial del propio estudiante, con la realización de una propuesta de indagación basada en la dinamización de experiencias previas, a través de comunidades de aprendizaje móviles (WhatsApp) y tutorías virtuales con los educandos involucrados. La finalidad es alcanzar el máximo de conocimientos previos que permitan interactuar con los materiales didácticos en distintos formatos, y su aplicación en la resolución de actividades, tareas, ejercicios, análisis, entre otros, de manera activa y colaborativa.

Las situaciones problemáticas indicadas anteriormente, así como su justificación respectiva, permiten plantear las siguientes preguntas de investigación: ¿Se verifica, en los participantes de los cursos en línea, si resulta más efectivo la construcción de conocimientos nuevos sobre la base de los saberes adquiridos previamente? ¿Se determina, si con el empleo de una comunidad de aprendizaje móvil en WhatsA$\mathrm{pp}$, se fomenta la interacción dinámica entre el mediador y los estudiantes, de manera que las actividades que se realizarán conjuntamente, se conviertan en una oportunidad para la creación de un nivel alto de aprendizaje, como resultado de dicha interacción?
¿Se precisa si la mediación pedagógica desempeña un papel primordial, en el adecuado rendimiento de los participantes dentro de un curso E-Learning? ¿Se demuestra que la tutorización en línea, empleada como medio de orientación, guía y realimentación por parte del tutor, resulta una estrategia clave que garantice la obtención de los conocimientos necesarios en los estudiantes?

Todas estas interrogantes menores podrán dar respuesta a una mayor: ipueden los facilitadores de cursos en línea, idear comunidades de aprendizaje móvil que permitan a la mayoría de los participantes, la adquisición de un nivel alto de conocimientos para la resolución de actividades planteadas en el aula virtual?

\section{Materiales y Métodos}

La realización de esta experiencia educativa implicó la utilización del procedimiento propuesto por Kemmis (1989) y Latorre (2003) con el fin de proceder con un estudio bajo la modalidad de investigación- acción, que tiene en cuenta aspectos de mejoras y cambio social. Se trata de planificar una acción, actuar o llevarla a la práctica, observar la misma y por último, reflexionar sobre lo ocurrido. Con base en el diseño instruccional del curso, se procedió con la puesta en marcha de una serie de fases o etapas que se describen a continuación:

Fase de planificación Se diseñó un curso en línea para los profesores de la UPEL -IMPM extensión Táchira; se preinscribieron sesenta (60) participantes e iniciaron el curso, cuarenta (40) de ellos. El ambiente virtual de aprendizaje fue diseñado con un entorno iconográfico, con estándares de accesibilidad, y contenidos sobre competencias TIC para docentes de la UNESCO, además de la matriz de competencia TIC del MEN-Colombia. La estructura instruccional de este curso se basó en la comprensión del diseño (UnderstandingbyDesign), contrastada con una propuesta inversa como el modelo ADDIE, propuesto por ESVI-AL, con el fin de evitar vacíos en el diseño del curso virtual.

Todo lo anterior se concibió para aprender a aprender in situ, en un entorno personal de aprendizaje (PLE) que para Atwell (2007) citado por Castañeda y Adell (2013, p. 186) implica que los PLE son "...un nuevo enfoque de cómo usar las tecnologías como medio de apoyo para el aprendizaje" sino que constituyen un nuevo enfoque para "aprender a aprender", Castañeda y Adell (2013, p.12). Donde "lo más importante son las personas", ya que se relacionan con otros (PLN Personal Learning Network), Por esto se infiere que se está aprendiendo al conectarse de manera informal y a través de una red personal de aprendizaje (PLN) a partir del PLE. Dentro del proceso de planificación, se llevó a cabo el método de trabajo siguiente: 
Se diseñaron unidades temáticas. Se creó una comunidad de aprendizaje móvil con la aplicación WhatsApp, para llevar a cabo las discusiones grupales dirigidas por el tutor, con el fin de que los participantes, a través del diálogo y las interacciones, adquirieran un nivel alto de conocimientos previos, que les permitieran afrontar las actividades planteadas en cada unidad del curso. Se creó, en paralelo, otra comunidad de aprendizaje en Facebook, con el fin de permitir la accesibilidad a otras discusiones. Se creó un Hashtag en Twitter y un espacio para las interacciones síncronas a través de Hangouts (Google + ) para la tutoría de los participantes.

Cada discusión grupal efectuada en la comunidad de aprendizaje móvil, fue planteada como desafío problematizador, afirmaciones, con citas de autores, entre otros, tomando en consideración una perspectiva constructivista-dialógica con realimentación de doble vía en forma continua.

Una vez terminada cada discusión grupal, se dieron a conocer las narrativas digitales de dicha discusión, las cuales se exhibieron en distintos artefactos digitales como Slideshare, con el fin de que los participantes pudieran leerlas en línea o las descargaran.

Fases de acción y observación. De acuerdo con Kemmis (1989), se exponen las etapas que corresponden a la puesta en marcha del plan elaborado, así como la observación. Las actividades se iniciaron en la cuarta semana del mes junio de 2016.

El aprendizaje fue totalmente constructivista, dialógico, social, informal, colaborativo, entendiendo la importancia de las conexiones para construir conocimiento, a través de las interacciones para la obtención de los conocimientos previos, que permitan afrontar los contenidos y actividades relacionadas con lo dado en cada unidad temática.

Las discusiones se iniciaron con planteamientos problematizados, con la idea de aplicar el principio de primero problematizar y después explicar, para promover el interés por la temática. Se comenzó problematizando, no explicando, en las distintas unidades; se hizo la discusión grupal en relación con las unidades, tales como: características del profesor del siglo XXI; metáforas del docente 2.0; modelo TPACK para planificar actividades con las TIC; aula invertida (FlippedClassroom); herramientas digitales para el aprendizaje visual; aprendizaje activo, colaborativo y herramientas web 2.0, con el fin de hacer una revisión colectiva de herramientas web 2.0.

Todas estas actividades estuvieron centradas en lo que los estudiantes deseaban hacer y, en la medida que se involucraban, se iban obteniendo resultados favorables.
Es necesario acotar que, muchas veces, los participantes entraban tarde a la discusión grupal, leían lo que se debatía a través de los aportes de cada uno de los miembros y se incorporaban en dicha discusión, por medio de sus dispositivos móviles y la aplicación WhatsApp. Luego de hacer un análisis del aprendizaje dentro del aula virtual del curso, se propuso la tutoría en línea para aquellos participantes con actividades atrasadas, donde se tomó en cuenta el problema 2 Sigma (tutoría) de manera síncrona, para que los que tenían menos de dos (2) actividades, pudieran superarse.

Fase de reflexión. En esta fase para el análisis y reflexión, se fueron dando respuestas a los objetivos de investigación. A través del primero de ellos, se intentó verificar en los participantes del curso La web: un espacio para el aprendizaje, la eficiencia en la construcción de conocimientos nuevos sobre la base de los saberes previamente adquiridos, pudiéndose apreciar que la experiencia proporcionada a través de la comunidad de aprendizaje móvil, para la mayoría de los profesores universitarios participantes, fue una experiencia significativa. Ellos desconocían que se podían aplicar estrategias por medio de discusiones grupales socializadas, a través de la aplicación WhatsApp como comunidad de aprendizaje móvil, con la aplicación de evaluación diagnóstica o formativa.

Ahora bien, en referencia con lo anterior, se precisa que en el proceso de construcción, desarrollo y fortalecimiento de conocimientos previos en las diferentes temáticas, estos se alcanzaron en un grado alto. Los aportes y análisis fueron sustanciosos para la generación de conocimientos, como punto de partida en la transformación de las prácticas dentro de los ambientes virtuales de aprendizaje o mediados por tecnologías. A continuación, se presentan extractos de las interacciones de una de las diferentes discusiones grupales a saber:

A.C.: Buenas noches...usualmente cuando se piensa en un espacio de aprendizaje digital se focaliza la atención en los recursos tecnológicos, los equipos, aparatos, hardware, software...

C.G.V.: ¿por qué? ¿Cómo adaptamos a los espacios digitales?

A.C.: Es la primera asociación, pero particularmente considera que debe pensarse la cultura, el clima humano, lo relacional, las actitudes y disposición de las personas que se encuentran vinculadas a esos ambientes o espacios.

C.G.V.: Así es mi estimado profe A.C., pero ya en la escuela tenemos el modelo 1:1

R.: Crear vínculos, una cultura colaborativa...pero una debilidad del aula 1.0 fue que no se pensó en la persona humana y se creyó que la incorporación "per se" de la "tecnología" solucionaría todo. Hubo iniciativas interesantes pero sin suficiente fundamentación pedagógica y didáctica. 
C.G.V.: grave error que lo estamos pagando. Al dotar al salón... más no a lo humano.

N.B.: Desde un enfoque sistémico; creo que Santana tiene razón que se podrían adaptar espacio de aprendizaje digital

N. J.: Todavía no intervengo... me falta revisar el material...

A.C.: Yo no lo he revisado pero me "lancé al ruedo".

C.G.V.: las discusiones grupales son algo previo a la temática, las mismas se orientan para llegar a la comprensión de la unidad en cuestión, así que intervenga profesor N.J.

N.J.: El ambiente de un espacio de aprendizaje digital en la web 2.0 debería tomar en cuenta (y adaptar según la necesidad y contexto) los elementos físicos mesas, sillas y paredes. Docente y alumno usan ambientes digitales en ambientes físicos probablemente distintos al aula.

C.G.V.: y cómo generamos un ambiente cómodo y agradable en la web.

N.C: En todo caso, el ambiente tecnológico, virtual siempre estará condicionado por los entornos reales.

Con las muestras de las intervenciones de los participantes, en los párrafos anteriores, se determinó que con el empleo de una comunidad de aprendizaje móvil en WhatsApp, se fomenta la interacción dinámica entre el mediador y los estudiantes. Ello permite que las actividades que se realizan conjuntamente, se conviertan en una oportunidad para la creación de un nivel alto de aprendizaje como resultado de dicha interacción.

\section{Resultados y Discusión}

En las interacciones realizadas dentro de la comunidad de aprendizaje móvil, se precisó que la mediación pedagógica fue primordial, puesto que permitió orientar y regular las discusiones sobre los temas planteados, así como dar recomendaciones para garantizar la contribución de los participantes.

Ello permitió que los participantes expusieran, de manera consistente, sus puntos de vista, con lo cual clasificaron los conocimientos previos para interactuar con el material didáctico, situación que sirvió para el desempeño en el rendimiento de los discentes dentro del curso e-Learning.

Esa mediación en función tutorial del facilitador, posibilitó la generación de conocimientos nuevos, al adaptar y actualizar in situ la información distribuida por el grupo de estudiantes, para ponerla al alcance de todos.
Las tecnologías o plataformas virtuales, por sí mismas, no tienen trascendencia si no existe una mediación ola aplicación de un aprendizaje adaptativo en el entorno, por medio de la tutoría personalizada o en grupos pequeños.

Quedó demostrado así y dio respuesta a lo formulado en el cuarto y último objetivo de investigación, de que la tutorización en línea empleada como medio de orientación, guía y realimentación por parte del tutor, resulta una estrategia clave que garantiza los conocimientos necesarios en los estudiantes o participantes; asimismo, que exista una comunicación recíproca e interactiva durante la tutoría, respalda el desarrollo del proceso formativo.

\section{Algunas opiniones de los participantes al respecto:}

Participante A.H.E.R. (UPEL-IMPM Táchira):....al tutor profesor C.G.V. quien me acompañó, motivando siempre a seguir, sus orientaciones sirviendo de guía, en esta experiencia de aprendizaje significativo para mí. ... se me presentaron algunos inconvenientes que me limitaron estar al ritmo de todos.

Participante L.B. de T. (UPEL-IMPM):... Por un momento creí no lograrlo pero la motivación, la afectividad y la asertiva comunicación por parte de nuestro profesor C.G.V. fueron pieza fundamental. Sin duda alguna el trabajo colaborativo también jugó un papel importante.

Participante L.A.G.A. (UNA-Centro Local Táchira). Referente al proyecto "La web un espacio para el aprendizaje" considero que es acorde a las necesidades constantes de innovación para quienes pretendemos mejorar nuestra praxis. Creo que se conformó una comunidad de aprendizaje. En cuanto al facilitador, desarrolló un proceso de mediación pedagógica excelente, giró instrucciones claras y precisas, estimuló la motivación de los participantes. El feedback fue oportuno.

Participante A.Y.G. (UPEL-IMPM, Sede Central). Mi opinión es un curso orientado a profesionales del sector aunque su estructura de contenidos lo hace apto para distintos niveles de conocimientos previos. El curso permite diferentes niveles de profundización; tanto puede servir para adquirir conocimientos generales sobre la calidad de la información y para profundizar sobre aspectos más concretos, la actitud de los profesores ha sido de plena disponibilidad y colaboración permanente. $\mathrm{Su}$ compromiso es el de responder cualquier cuestión en cualquier momento que el participante lo necesitara. La sensación que he tenido es que la comunicación ha funcionado bien, posibilitando un flujo de trabajo ágil...., al Profesor C.G.V. y a la Dra. R.R. por su disposición al trabajo, su capacidad de respuesta, su dedicación y sobre todo dominio y claridad de los temas que allí se trataron. 
Comunidades de aprendizaje móvil, MasteryLearning y el Problema 2 sigma como estrategias para el conocimiento previo, en la resolución de actividades $\mathbf{3 1}$

Participante N.B. (UPEL-IMPM Sede Central)... encontramos: instrucciones precisas, va desde actividades simples hacia las más complicadas, es atractivo visualmente, genera expectativas de aprendizaje, obliga a centrarse en lo que se hace, la interacción es fluida, se respetan los procesos y tiempos individuales, así como ofrece realimentación constante sobre las actividades.... su comprensión de los participantes no es tarea sencilla, exige dedicación, horas de trabajo, conocimiento y manejo profundo del tema...

Participante L.J.P. (UPEL-IMPM YARACUY):“... El proyecto es sin lugar a dudas EXCELENTE, la experiencia adquirida básicamente con esta "comunidad" me ha permitido interactuar... para comentar, compartir, logrando obtener un crecimiento gracias al aprendizaje interactivo que reflejan lo importante que ha sido para lograr mejorar nuestro desempeño docente... Considero brillante la constante participación del Profesor nos guio a conocer y manejar las novedades de un entorno en constante renovación para estar en contacto con el mundo digital."

\section{Conclusiones.}

La implementación de prácticas pedagógicas adecuadas puede llevar al estudiante a la participación, colaboración, cooperación y reflexión, a través de interacciones entre pares (el mediador y los contenidos didácticos), cruciales para el proceso de enseñanza y aprendizaje al internalizar conceptos, ideas y contenidos nuevos que faciliten su acomodación respecto con los conocimientos previos.

La mediación del profesional de la docencia es imprescindible para el buen desarrollo de las actividades planteadas; además, establece tutorización, interacciones entre estudiante-estudiante, tutor-estudiante y viceversa. Por otra parte, motiva y guía las relaciones interpersonales del grupo, plantea preguntas apropiadas para sugerir al estudiante, autonomía, responsabilidad, autoaprendizaje, creatividad y confianza al encontrarse dentro de una comunidad de aprendizaje digital, porque la adquisición de la información también está sujeta a la interacción social.

De igual manera, la medición, recopilación, análisis y presentación de datos sobre los estudiantes, dentro del contexto y las interacciones que allí se generaron, hizo posible que se comprendieran las necesidades del proceso de aprendizaje que se estaban desarrollando.

Se optimizaron los entornos en los que se produjo, para evaluar estrategias dirigidas a aquellos estudiantes que estaban atrasados; un elemento clave, estuvo en la comunicación a través de los distintos canales de intercambio o enlace, proporcionados dentro del ambiente virtual de aprendizaje.
Por otra parte, la analítica de aprendizaje permitió verificar el desempeño de los estudiantes, con lo cual el mediador del proceso pudo aplicar estrategias nuevas, tales como nueva tutorización (problema 2 sigma) a los participantes que tenían menos de dos (2) actividades, con lo que se obtuvo $85 \%$ de recuperación para la finalización exitosa del curso.

Es necesario indicar además que, al aplicar el diseño de MasteryLearning (dominio del aprendizaje) apoyado en la estrategia del problema 2 sigma, se logró una mayor comprensión de los contenidos al tener un $90 \%$ de conocimientos previos para afrontar y realizar las actividades planteadas.

Se hace pertinente denotar que, en el curso La web: un espacio para el aprendizaje (edición UPEL-IMPM Táchira 2016), se logró integrar el aprendizaje personalizado, adaptativo y flexible en los entornos de aprendizaje digitales, el aprender-aprender y la flexibilidad en la educación superior que van unidos a la formación continua a lo largo de la vida.

Se consiguió a través de la red personal de aprendizaje generada en las distintas comunidades de aprendizaje preparadas para tal fin, tales como Facebook y Twitter. Se puede afirmar que, la conexiones sociales dadas por los distintos canales de comunicación síncrona y asíncrona, continúan siendo la clave para el aprendizaje.

\section{Referencias}

Asociación Universitaria de Formación del Profesorado, AUFOP. (Abril, 2010). Revista interuniversitaria de formación del profesorado, (67).

Asociación Universitaria de Formación del Profesorado, AUFOP. (Abril, 2010). Revista interuniversitaria de formación del profesorado, (67).

Block, J. H. y Anderson, L. W. (1975). Mastery Learning in Classroom Instruction.New York: MacMillan.

Bloom , B. (1968).El aprendizaje para el dominio (Learning for Mastery). UCLA-CSEIP.

Bloom , B. (1984). The 2 Sigma Problem: The Search for Methods of Group Instruction as Effective as One-to-One Tutoring. Educational Researcher. Recuperado de http://web.mit.edu/5.95/readings/bloom-two-sigma.pdf.

Castañeda , L. y Adell, J. (2013).Entornos personales de aprendizaje: claves para el ecosistema educativo en red. Recuperado de http://www.um.es/ple/libro/.

Fidalgo , Ángel. (2014).Comunidad de aprendizaje. Recupera do de https://innovacioneducativa.wordpress.com/2014/01 /26/comunidades-de-aprendizaje/. 
Farnos , Juan. (2015). De Mastery Learning al PersonalizedLearning! (Educación Disruptiva). Recuperado de https://juandomingo farnos.wordpress.co $\mathrm{m} / 2015 / 09 / 13$ / del master y - learning-al- personalized - learning - educacion-disruptiva/.

García F., N. (2002). Sistema de trabajo con las Tics en el sistema educativo y en la formación de profesionales: la comunidad de aprendizaje. Recuperado de http://www.um .es/ead/red/6/comunidades.pdf.

González , R.M. (2015) Motivación para la "inclusión”, características y necesidades de los estudiantes y docentes en la universidad. (Aplicativo con análisis de gráficos estilísticos). Eco.Mat. 2015; 6(1): 107-115. Recuperado de http://revistas.ufps.edu.co/ojs/index.php/ecomatematico/article/view/464/481.

Kemmis , S. (1989).Investigación en la acción. Barcelona: Ediciones Graó. España.

Latorre , Antonio (2003).La investigación - acción: conocer y cambiar la práctica educativa. Recuperado de https://books.google.co.ve/books?id=e1PLxGcRf8gC\&printsec $=$ frontcover $\# \mathrm{v}=$ onepage $\& \mathrm{q} \& \mathrm{f}=$ false.

Meza , J. (2012). Modelo pedagógico para proyectos deformación virtual. Ministerio federal de cooperación económica y de desarrollo. Recuperado de https:/gc21.giz.de/ibt/var/ap p/w p 342 P / $1522 /$ w p - content / u ploads/2013/02/Ebook-final.pdf.

Ontoria , A. y otros (2006). Mapas conceptuales. Una técnica para aprender. (13 $3^{\mathrm{a}}$.ed.). Madrid: Narcea, S.A. de ediciones. final.pdf.

Pabón - Gómez Jorge. (2014). Las Tics y la lúdica como herramientas facilitadoras en el aprendizaje de la matemática. Eco.Mat. 2014; 5(1): 37-48. Recuperado en http://revistas.ufps.edu.co/ojs/index.php/ecomatematico/ article/view/62/76.

Pacheco -Carrascal, N. (2016) "La motivación y las matemáticas", Eco.Mat. 2016; 7(1): 149-158. Recuperado dehttp://revistas.ufps.edu.co/ojs/index.php/ecomatematico/aricle/view/1026/1060.

Pérez, A (2009). La comunicación y la interacción en contextos virtuales de aprendizaje. Apertura. Recuperado de http://www.udgvirtual.udg.mx/apertura/index.php/apertu$\mathrm{ra} / \mathrm{rt} /$ printerFriendly/15/18.

Piaget (1968) Educación e instrucción. Buenos Aires: Proteo.

Siemens, G. (2004). Connectivism: a learning theory for the digital age. Recuperado de http://www.elearnspace.or$\mathrm{g} /$ Articles/connectivism.htm.
Vygotsky . (1978). El método instrumental en psicología. Krúpskaia. 